\section{Discussion}

Neurological complications in thalassaemia are rare. Extramedullary haematopoiesis is well recognised in thalassaemia at sites such as the liver and the spleen. Rarely such haematopoietic tissue may be found within the spinal canal, extradurally, causing compression of the spinal cord. ${ }^{1-3}$ Infrequently, infiltration of the middle ear by haematopoietic tissue may lead to hearing loss. ${ }^{4}$ These complications are more common on a low transfusion regime because it leads to bone marrow hyperplasia with widening of bones and extramedullary haematopoiesis. ${ }^{3}$ This patient was on a low transfusion regime due to poor compliance and had radiological evidence of widening of bones.

The commonest cause of an isolated unilateral lower motor neurone facial nerve palsy at this age is Bell's palsy; however, in about $85 \%$ of cases, resolution occurs with time. Absence of evidence of middle ear infection, or a vesicular eruption around the external auditory meatus at the time of the facial palsy, excludes otitis media and herpes zoster as likely causes. Poliomyelitis or another enteroviral infection as a potential cause is unlikely in this patient as she had received appropriate doses of oral polio vaccine in the past and also because the two nerve lesions were spaced out in time. Therefore, the probable cause for the facial nerve palsy is pinching of the nerve at one of the narrow points along its course, such as within the facial canal, due to widening of diploeic bone. The radiological evidence of widening of skull diploe supports this hypothesis, although an unresolved Bell's palsy cannot be conclusively ruled out.

The reason for the phrenic nerve palsy is more difficult to explain. It may have been due to pressure from a small paravertebral mass of haematopoietic tissue. Although irradiation has been used successfully to treat paravertebral masses, ${ }^{2}$ it could not be used in this patient because exact localisation was not possible.

\footnotetext{
References

${ }^{1}$ Cross JN, Morgan OS, Gibbs WN, Cheruwanky I. Spinal cord compression in thalassaemia. J Neurol Neurosurg Psychiatry 1977;40:1120-2.

${ }^{2}$ Issaragrisil S, Piankigagum A, Wasi P. Spinal cord compression in thalassaemia. Report of 12 cases and recommendations for treatment. Arch Intern Med 1981;141:1033-6.

3 Anonymous. Spinal cord compression in thalassaemia. [Editorial]. Lancet 1982;i:664-5.

${ }^{4}$ DeVirgiliis S, Argiolu F, Sanna G, et al. Auditory involvement in thalassaemia major. Acta Haematol (Basel) 1979;61:209-15.
}

Correspondence to Professor SP Lamabadusuriya, Department of Paediatrics, Faculty of Medicine, University of Ruhuna, Galle, Sri Lanka.

Accepted 14 November 1988

\title{
Betaine for treatment of homocystinuria caused by methylenetetrahydrofolate reductase deficiency
}

\section{E HOLME, ${ }^{*}$ B KJELLMAN, $\dagger$ AND E RONGE $\dagger$}

${ }^{*}$ Department of Clinical Chemistry, Gothenburg University, and †Department of Paediatrics, Kärnsjukhuset, Skövde, Sweden

SUMmaRY A 24 day old girl with homocystinuria and hypomethioninaemia caused by methylenetetrahydrofolate reductase deficiency presented with rapidly progressing encephalopathy and myopathy. An almost complete recovery was achieved by treatment with betaine.

5,10-Methylenetetrahydrofolate reductase (MTHFR) deficiency ${ }^{1}$ leads to deficient remethylation of homocysteine and is one of the causes of homocystinuria (figure). The other causes of homocystinuria are defects in cobalamin metabolism or deficient cystathionine $\beta$-synthase activity.
Methionine accumulates with homocystine in cystathionine $\beta$-synthase deficiency, ${ }^{1}$ whereas in patients with disorders in cobalamin metabolism or with MTHFR deficiency methionine synthase is functionally deficient and the increased concentration of homocystine occurs with normal or decreased methionine concentrations. In the latter conditions neurological impairment is the most important clinical finding. When symptoms occur in early infancy there is often rapid deterioration with respiratory failure.

Methylmalonic aciduria or macrocytic or megaloblastic changes of the bone marrow, or both, usually occur with homocystinuria in patients with disorders of cobalamin metabolism. These condi- 


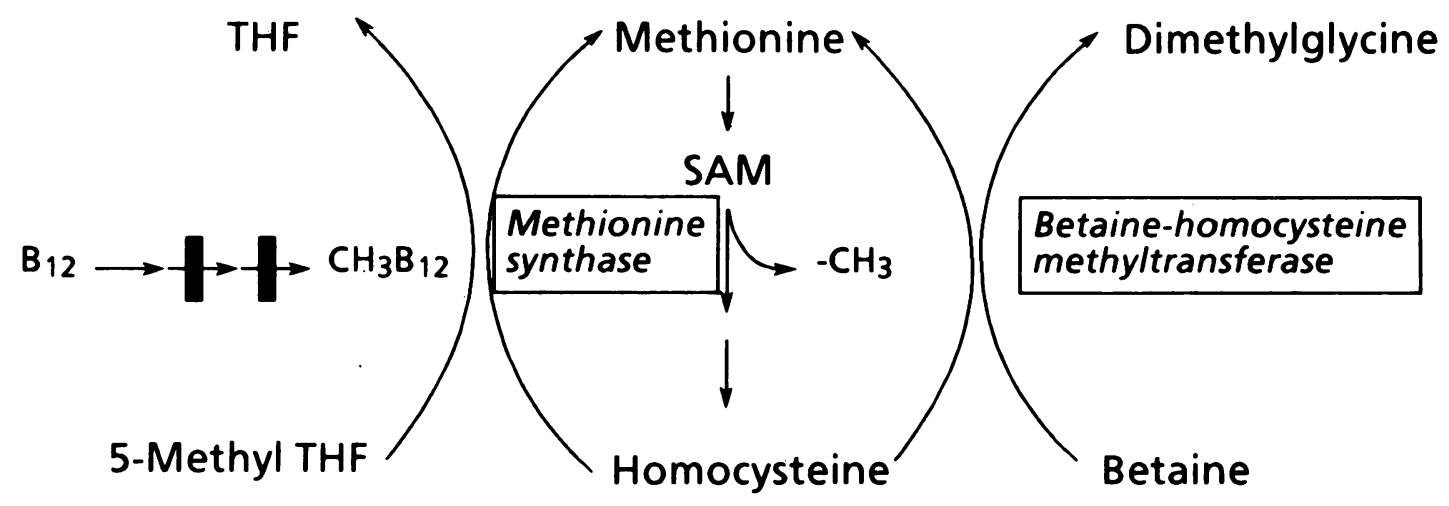

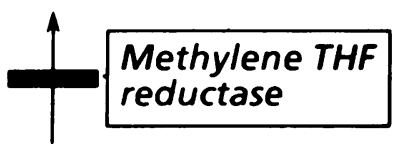

5,10-Methylene THF

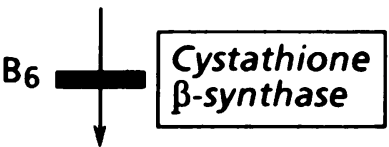

Cystathionine

Figure Metabolic pathways of homocysteine. Sites of metabolic defects leading to homocystinuria are indicated by blocks. $S A M=S$-adenosylmethionine; $T H F=$ tetrahydrofolate; $B_{12}=$ cobalamin; and $B_{6}=$ pyridoxal 6-phosphate.

tions usually respond to high doses of hydroxocobalamin. In MTHFR deficient patients, treatment with folate or cobalamin does not increase the methionine synthase activity as shown by the poor biochemical and clinical response. ${ }^{2}$

The alternative to the methyltetrahydrofolate dependent remethylation pathway of homocysteine is the betaine dependent pathway (figure). Treatment with betaine was efficient in lowering homocystine concentrations and reduced methionine concentration to normal in a 2 year old child with MTHFR deficiency. ${ }^{3}$ The child was microcephalic and severely retarded when the betaine treatment was started, and the clinical response was limited. Two other patients treated with betaine have been reported (NJ Brandt, et al. Treatment of methylenetetrahydrofolate reductase deficiency from the neonatal period. Presented at the Society for the Study of Inborn Errors of Metabolism Annual Symposium, Amersfoort, The Netherlands, 1986). One was diagnosed prenatally. Betaine was started at the age of 2 weeks before any symptoms had appeared, and at the age of 15 months psychomotor development was normal. In the other child, treatment was started 27 days after the onset of symptoms at the age of 36 days. There was 'considerable developmental improvement' by 12 months of age. Two infants who died after 4 days and after 3 weeks of treatment have also been mentioned in a review. ${ }^{2}$ We now report an infant in whom successful treatment with betaine was started at the age of 7 weeks after a period of three weeks during which there was rapid progressive neurological deterioration.

\section{Case report}

A baby girl, the first child of healthy parents who are cousins with no family history of neurological, psychiatric, or thrombovascular disease, was born after a normal pregnancy. Vacuurm extraction was used because of slow progression of the delivery. Apgar scores were 4, 5, and 8 at one, five, and 10 minutes. The birth weight was $3030 \mathrm{~g}$ and the head circumference $34 \mathrm{~cm}$. Mild respiratory distress lead to two days treatment in an incubator. At 6 days of age the child was discharged from hospital. She was breast fed and remained healthy until 24 days of age, when she had an attack of cyanosis and unconsciousness. On admission she was pale, hypotonic, and lethargic. Blood glucose and serum electrolyte concentrations, acid base balance, and analysis of cerebrospinal fluid were normal. The haemoglobin concentration was $136 \mathrm{~g} / \mathrm{l}$. C reactive protein concentration was less than $10 \mathrm{mg} / \mathrm{l}$.

Electrocardiogram and chest radiograph were normal. Two electroencephalograms obtained during the first week after admission were normal, although weak muscular twitching, tongue fasciculation, and seizures developed.

A positive urinary nitroprusside reaction was found two days after admission. Quantitative anino 
acid analysis by ion exchange chromatography showed a high plasma homocystine concentration and no detectable methionine. Increased urinary excretion of homocystine and cystathionine were also found. Urinary organic acid excretion analysed by gas chromatography and mass spectrometry was normal. The bone marrow morphology was normal. Plasma cobalamin and erythrocyte folate concentrations were also normal.

Treatment with pyridoxine $(100-150 \mathrm{mg} /$ day $)$, hydroxocobalamin ( $1 \mathrm{mg} /$ day), and folic acid (30-60 $\mathrm{mg} /$ day) was started. There was no response to the treatment, her clinical condition deteriorated rapidly, and respiratory failure necessitated assisted ventilation on the 21 st day after admission. The electroencephalogram was then abnormal showing abundant spike and wave potentials. After three days of artificial ventilation betaine ( $3 \mathrm{~g} /$ day) and methionine $(0.5 \mathrm{~g} /$ day $)$ were given orally. After two days of treatment the muscular tone improved, and after two weeks there was no further need for assisted ventilation. The homocystine concentrations in urine and plasma decreased below the detection limit and a normal plasma methionine concentration was obtained without methionine supplementation (table). The electroencephalogram returned to normal, and she caught up in growth and psychomotor development.

MTHFR deficiency was established by measurements in cultured skin fibroblasts as described by Mudd et $a l .{ }^{4}$ The activity found was $0.34 \mathrm{nmol} /$ (hour $\times$ mg protein). The mean activity in five controls was $15.5 \mathrm{nmol} /($ hour $\times \mathrm{mg}$ protein) (range 9.0-32).

Total plasma homocysteine concentration-that is, the combined free and protein bound fractions, was $78 \mu \mathrm{mol} / 1$ (reference interval in adults $4 \cdot 2-17 \cdot 8$ $\mu \mathrm{mol} / \mathrm{l})$ at the age of 9 months, when she was taking $3 \mathrm{~g}$ betaine daily. The daily intake of betaine was then increased to $6 \mathrm{~g}$ daily. The effect on total homocysteine concentration was limited; after two months total homocysteine concentration had only decreased to $70 \mu \mathrm{mol} / \mathrm{l}$, but the plasma methionine concentration though still within the normal range, had increased.

Mainly because of persisting feeding difficulties the child was kept in hospital until the age of 5 months. When last seen at the age of 12 months the increments of length, weight, and head circumference were normal, following the 50th, 25th, and 10th percentiles, respectively. Her psychosocial behaviour was normal but her motor development was retarded by two to three months.

\section{Discussion}

Methylenetetrahydrofolate reduction is the first step in the biosynthesis of methyl groups, whichthrough homocysteine methylation and S-adenosylmethionine formation-participate in phospholipid biosynthesis and RNA and DNA modification, among other things.

As shown by the return to normal of plasma and urinary methionine and homocysteine concentrations in our case as in others, (NJ Brandt, et al. Treatment of methylenetetrahydrofolate reductase deficiency from the neonatal period. Presented at the Society for the Study of Inborn Errors of Metabolism Annual Symposium, Amersfoot, The Netherlands, 1986), ${ }^{2-3}$ exogenous betaine can supply methyl groups for homocysteine methylation. The accompanying clinical recovery in our case indicated that the metabolic derangement (presumably a shortage of methyl groups) responsible for the neurological symptoms was corrected by betaine treatment. Thus is is imperative to recognise these patients early so as to start treatment before irreversible damage has occurred.

Table Clinical and biochemical responses to different treatments

\begin{tabular}{|c|c|c|c|c|c|c|c|}
\hline & \multicolumn{7}{|c|}{ Age (weeks) } \\
\hline & 4 & 5 & 8 & 9 & 21 & 29 & 36 \\
\hline & \multicolumn{2}{|c|}{ Deterioration } & \multicolumn{2}{|c|}{ Improvement } & & & \\
\hline \multicolumn{8}{|l|}{ Treatment: } \\
\hline Pyridoxine, hydroxocobalamin & No & Yes & Yes & Yes & Yes & No & No \\
\hline Methionine $500 \mathrm{mg} / \mathrm{day}$ & No & No & Yes & No & No & No & No \\
\hline Betaine $3 \mathrm{~g} /$ day & No & No & Yes & Yes & Yes & Yes & Yes \\
\hline Plasma methionine concentration $(\mu \mathrm{mol} / \mathrm{l})$ & $<10$ & $<10$ & 395 & 15 & 18 & 18 & 18 \\
\hline Plasma homocystine concentration $(\mu \mathrm{mol} / \mathrm{l})$ & 15 & 30 & $<10$ & $<10$ & $<10$ & $<10$ & $<10$ \\
\hline \multicolumn{8}{|l|}{ Urinary homocystine concentration } \\
\hline (mmol/mol creatinine) & 230 & 140 & - & 18 & 17 & $<10$ & $<10$ \\
\hline
\end{tabular}


To ensure long term health, betaine should if possible be given in doses high enough to return total plasma homocysteine concentration to normal, as even a moderate increase in a possible risk factor for thrombovascular disease. ${ }^{5}$

We thank Dr B Hultberg, Department of Clinical Chemistry, University of Lund, for determining the total plasma homocysteine concentrations. This study was supported by grant $13 \mathrm{X}-585$ from the Swedish Medical Research Council and the KSS Paediatric Research Foundation.

\section{References}

${ }^{1}$ McKusick VA, ed. Mendelian inheritance in man. Catalogs of autosomal dominant, autosomal recessive, and x-linked phenotypes. 7th ed. Baltimore: Johns Hopkins University Press, 1986.
${ }^{2}$ Erbe RW. Inborn errors of folate metabolism. In: Blakely RL, ed. Folate and pterins. Vol 3. Boston: John Wiley, 1986: 413-65.

3 Wendel U, Bremer HJ. Betaine in the treatment of homocystinuria due to 5,10-methylenetetrahydrofolate reductase deficiency. Eur J Pediatr 1984;142:147-50.

${ }^{4}$ Mudd SH, Uhlendorf BW, Freeman JM, Finkelstein JD, Shih VE. Homocystinuria associated with decreased methylenetetrahydrofolate reductase activity. Biochem Biophys Res Commun 1972;46:905-12.

5 Brattström LE, Hardebo JE, Hultberg BL. Moderate homocysteinemia - a possible risk factor for arteriosclerotic cerebrovascular disease. Stroke 1984;15:1012-6.

Correspondence to Dr E Holme, Department of Clinical Chemistry, Gothenburg University, Sahlgren's Hospital, S-413 45 Gothenburg, Sweden.

Accepted 14 November 1988 